Accepted refereed manuscript of:

Bartie P, Mackaness W, Petrenz P \& Dickinson A (2015) Identifying related landmark tags in urban scenes using spatial and semantic clustering, Computers, Environment and Urban Systems, 52, pp. 48-57.

DOI: $10.1016 / j . c o m p e n v u r b s y s .2015 .03 .003$

(C) 2015, Elsevier. Licensed under the Creative Commons AttributionNonCommercial-NoDerivatives 4.0 International http://creativecommons.org/licenses/by-nc-nd/4.0/ 


\title{
Identifying Related Landmark Tags in Urban Scenes using Spatial and Semantic Clustering
}

\begin{abstract}
There is considerable interest in developing landmark saliency models as a basis for describing urban landscapes, and in constructing wayfinding instructions, for text and spoken dialogue based systems. The challenge lies in knowing the truthfulness of such models; is what the model considers salient the same as what is perceived by the user? This paper presents a web based experiment in which users were asked to tag and label the most salient features from urban images for the purposes of navigation and exploration. In order to rank landmark popularity in each scene it was necessary to determine which tags related to the same object (e.g. tags relating to a particular café). Existing clustering techniques did not perform well for this task, and it was therefore necessary to develop a new spatial-semantic clustering method which considered the proximity of nearby tags and the similarity of their label content. The annotation similarity was initially calculated using trigrams in conjunction with a synonym list, generating a set of networks formed from the links between related tags. These networks were used to build related word lists encapsulating conceptual connections (e.g. church tower related to clock) so that during a secondary pass of the data related network segments could be merged. This approach gives interesting insight into the partonomic relationships between the constituent parts of landmarks and the range and frequency of terms used to describe them. The knowledge gained from this will be used to help calibrate a landmark saliency model, and to gain a deeper understanding of the terms typically associated with different types of landmarks.
\end{abstract}

\section{Keywords:}

Urban landmarks, scene tagging, trigram, tag clustering, mereology, graph clustering

\section{Introduction}

Human Computer Interaction (HCI) continues to evolve, creating more natural interfaces that increase productivity for a wider audience across a range of use environments. In particular mobile devices, used while moving, are receiving a lot of attention in the post-desktop era (Daley, 2012). As a result of this shift, and with the increase in processing power and improved statistical language models, speech recognition has grown in popularity for interacting with mobile devices. Smartphone applications such as Siri (Apple) and Cortana (Microsoft) allow the user to book diary events, look up information, or ask for directions, using only speech input.

While automatic speech recognition has improved the interaction is not entirely natural as the application is unaware of the user's surroundings and unable to refer to things as people typically do in conversation, for example to comprehend a question such as "What's that statue over there?", or to direct the user to "the café next to the bridge". To include such environmental references these devices need to model their surroundings and refer to features in common ways, so that the interface can become so natural and intuitive it is not even noticed (Weiser, Gold, \& Brown, 1999).

It has been recognised for some time that further progress in mobile HCI will include expanding the machine's abilities to refer to objects in the user's surroundings, and to consider the context in which the device is being used (Bartie \& Mackaness, 2006; Chen \& Kotz, 2000; Long, Aust, Abowd, \& Atkeson, 1996; Noh, Lee, Oh, Hwang, \& Cho, 2012; Zipf, 2002). A key aspect of this link between virtual and real worlds is the use of common 
anchor points, or landmarks, which can be recognised and referred to by both the user and the machine. For example including a reference to a salient object when giving a navigation instruction. There are a number of challenges in doing this, which include having access to a complete dataset of objects with corresponding attribute and positional information, a method to identify landmark candidates from the dataset, and the ability to select the most suitable candidate for a particular task (e.g. the most suitable landmark for a turn instruction) (KaiFlorian Richter \& Winter, 2014).

As part of a wider research project looking at supplementing location based services with knowledge of the user's environment, and thereby offering a greater interaction between machine and place, a web based experiment was undertaken to collect data on what users considered to be landmarks in urban scenes and to understand better how they describe those objects. Participants were asked to identify features by viewing urban images and tagging those items they considered useful in forming navigation instructions, adding a text annotation to each feature that they tagged. In some cases users supplied tags for single object features (e.g. a statue), while in others a label was used to represent a collection of features, such as a castle with its many outbuildings and walls.

In order to determine the most salient objects in each scene the user generated tags first need to be grouped according to the object they referred to, so that the number of unique users could be calculated per landmark. The assumption was made that the more salient features would be tagged by a larger number of participants who considered it a suitable landmark for wayfinding. Such analysis would give a feature ranking, thus establishing the most dominant landmarks in each scene, and provide a better understanding of the importance hierarchy of features and sub-feature parts (e.g. the clock and the clock tower it is on). By establishing a landmark ranking in each scene the various input metrics for the saliency model could be adjusted so that a model's output more closely matched human landmark identification choices.

While spatial clustering methods can be used to highlight tag concentrations across the image, it did not offer adequate functionality to identify discrete objects, as tags in close proximity may relate to different real world objects which appear close merely because of the perspective view in the image. Therefore it was necessary to develop a clustering algorithm able to group tags based on both the spatial location of the tag as well as the supplied text label. The process was complicated by the range of descriptive terminology supplied in the labels. For example the same landmark may be described as a church by one participant, and as a clock tower by another referring to a subpart of the same structure. The algorithm developed used a statistical sentence matching technique to link tags with related nearby annotations, forming tag networks where nearby tags with similar content were considered to have a strong relationship.

The paper begins by explaining the background and motivation for this research, followed by a description of the web experiment conducted to collect data in Section 3, and then the issues encountered with generating landmark rankings based on spatial clustering and the need to develop a spatial-semantic clustering function, which is outlined in Section 5. The paper concludes with suggestions for deriving other outputs from the tag data using this clustering technique, and highlights some of the remaining issues which require future research. 


\section{Background and Motivation}

97

98

99

100

101

102

103

104

105

106

107

108

109

110

111

112

113

114

115

116

117

118

119

120

121

122

123

124

125

126

127

128

129

130

131

132

133

134

135

136

137

138

139

140

141

Landmarks are one aspect of the environment frequently referenced, as they assist in forming mental representations of space (Hirtle \& Heidorn, 1993; Tversky, 1993), and in wayfinding tasks (Caduff \& Timpf, 2008; Duckham, Winter, \& Robinson, 2010; Lovelace, Hegarty, \& Montello, 1999; Werner, Krieg-Bruckner, Mallot, Schweizer, \& Freksa, 1997; Winter, Tomko, Elias, \& Sester, 2008). Studies show that when exploring a new urban region people build a mental model of the space by firstly recognising landmarks, then over time these are joined together into sequences to form routes, which depending on the complexities of the space may lead to a more comprehensive model of the space known as survey knowledge (Hirtle \& Jonides, 1985).

Landmarks are defined as identifiable features in an environment, whose saliency may be calculated by comparing scores for particular attributes (e.g. their size) and identifying those which deviate from the mean (Elias, 2003; Elias \& Brenner, 2004; Raubal \& Winter, 2002). These are the objects unlikely to be confused with others, as they appear different to their surroundings (e.g. churches, statues) or are well known international brands (e.g. Starbucks, McDonalds). Landmarks are particularly useful when travelling to a new destination as they can be used at decision points to help orient the navigator, along routes to confirm the location, and as distant landmarks (Lovelace et al., 1999). While it is common for people to include landmarks in conversation, current smartphone digital assistant applications, such as Apple's Siri, Microsoft's Cortana, and Samsung's S-Voice, are unaware of the user's environment and therefore unable to refer to surrounding objects. As speech based interfaces continue to develop it will be useful to include better context awareness which can establish the user's environment and include references to visible landmarks around the user. Google's Project Tango (Lee, 2014) shares a similar ambition to enrich the user experience by allowing software to consider the world beyond the phone's hardware and to consider time and space at a more human scale. Such an ability would allow for the generation of more natural human computer interactions, helping to reduce the seam which exists between users and technology (Ishii, Kobayashi, \& Arita, 1994). Such situational awareness will extend the range and capabilities of mobile applications, as has already been demonstrated in prototype applications (Bartie \& Mackaness, 2006; Mackaness et al., 2014).

There are two parts to the process of using landmarks in forming navigation instructions, or in generating referring expressions to describe the location of city objects. These are the identification of suitable candidate landmarks from all known objects, and then determining the most appropriate candidate for a given task (e.g identifying the landmark which best supports a turning instruction in a wayfinding task) (Kai-Florian Richter \& Winter, 2014).

The task should determine which landmarks are selected according to the route taken rather than using pre-set items from a static list of landmarks in the region (Nothegger, Winter, \& Raubal, 2004). Similarly when using landmarks to describe a scene or direct the user's gaze, a selection process is required to determine the most suitable candidates from those in the current view. The ambition is to provide no more information than is necessary, according to Grice's maxim of quantity (Grice, 1975), and therefore the selection process should ensure a minimal set of highly relevant landmarks are returned. This goes beyond measuring the path of photons from the observer to the target feature, as it is not only a question of which are physically visible, but also which are noticed by an observer at that location. For example when asked to identify statues in Figure 1a people will often notice the statue of a black horse in the foreground but many fail to spot the more distant statue, as highlighted in Figure 
142 1b. This may be partly because it is further away, but perhaps it is also a factor of the 143 surrounding distractors in the scene of buildings and trees making it harder to separate 144 visually from background objects, or perhaps it is an artefact of the position of the statues in 145 the image frame.

a)

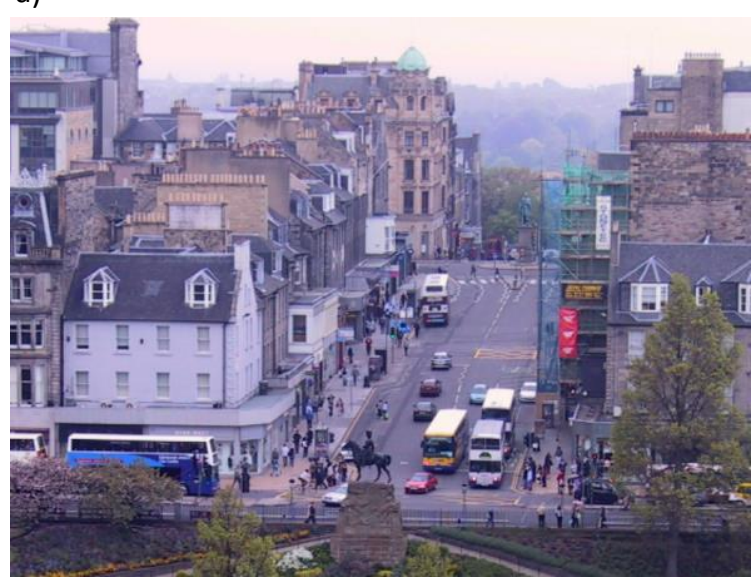

b)

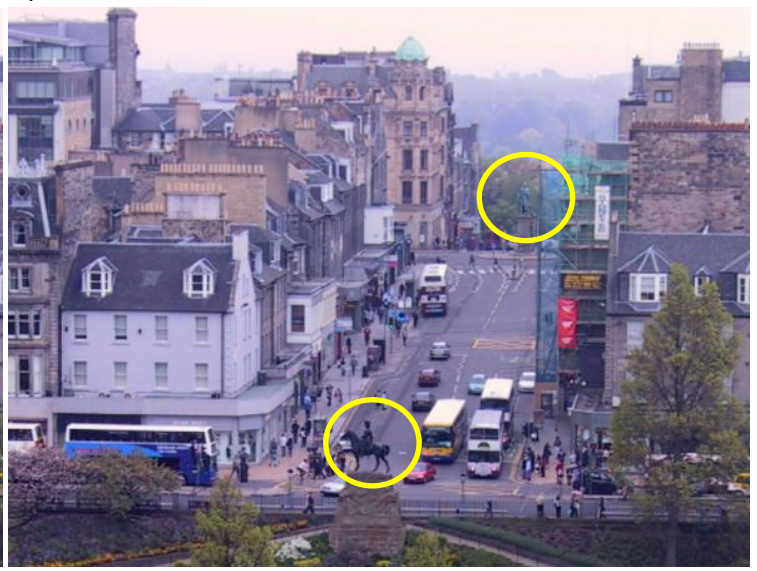

Figure 1: Visible objects are not always noticeable, with many people failing to notice the second more distant statue in this scene

147 Existing techniques to detect landmarks from photo collections using cluster-based 148 techniques consider each image as a single object to be classified. For example Papadopoulos 149 et al (2010) treats each image as a node in a graph, and exploits computer vision and user 150 tag similarity metrics to find corresponding images of landmarks or events. Other approaches 151 for automated and semi-automated image clustering (Vonikakis, Jinda-Apiraksa, \& Winkler, 152 2014; Wang, Ji, Tian, \& Hua, 2012) or the linking to location (Ahern, Naaman, Nair, \& 153 Yang, 2007) also consider the images as single objects. There are research efforts to assign 154 tags to features in images, the Tag-to-Region Assignment Problem (Liu, Hua, \& Zhang, 155 2011), to correspond to the semantic region within an image but these are not yet robust. For 156 this research the images were considered as a means to portray real world features to a web 157 audience, with a goal of extracting information from the participants about the real world 158 objects portrayed in the images. Therefore the tag annotations were supplied at an object 159 feature level rather than a request to more generally describe the entire image.

160 There are arguments for imposing structured vocabularies to enable greater semantic parsing 161 of supplied annotations (Tousch, Herbin, \& Audibert, 2012), however for this research 162 participants were permitted to enter any text without restrictions so that a wide range of 163 descriptors would be collected for analysis.

\section{3. Web Experiment}

166 A web based experiment was conducted in which human subjects were asked to identify landmarks in a number of urban scenes. The experiment was publicised through social media, attracting 185 participants. Users were assigned images randomly from a set of 37, and able to leave the experiment at any time but encouraged to complete as many images as 
possible by giving them an additional entry into a prize draw for each completed set. For each task the participant saw an image of part of Edinburgh city (Scotland), and was asked to identify what they considered to be landmark features by tagging them on the image. The user's profile and knowledge of the city was recorded as part of this process.

174 All images were captured on the same day in the early morning over a period of ninety minutes, in an effort to reduce object occlusion by other city occupants (e.g. buses, pedestrians) and to minimise weather and lighting variation. The ambition was to replicate as closely as possible the street experience, although it is recognised from previous landscape studies that imagery can introduce a bias in the way that it is captured and displayed (Daniel \& Vining, 1983; Linton, 1968; Shafer \& Brush, 1977; Zube, Sell, \& Taylor, 1982). In an effort to minimise these effects the images were captured using a wide angle lens, and as computer monitors do not offer the same level of visual detail as when on the street, a magnifying region was added to the web viewer, as shown in Figure 2. This allowed the participant to see a magnified portion of the image as they moved the mouse crosshair around the main image, giving a similar level of detail to that experienced on the street, and enabling them to more easily identify and tag more distant and smaller objects.

186 Once the participant had clicked on the image at the location of something they considered interesting, they were presented with an input box to enter free text which described the object (Figure 2b), such as a church, pub, or no entry sign. Each participant was permitted up to 12 tags per image, and asked to provide a short description for each tag. The tag limitation was imposed to encourage participants to limit their tagging to the most salient objects, and to then move on to the next image.

192

(a)

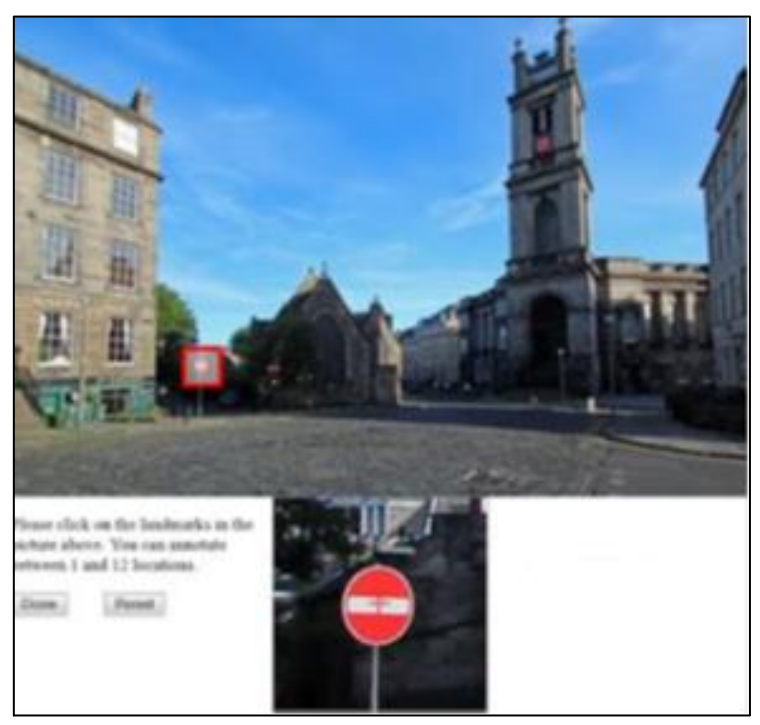

(b)

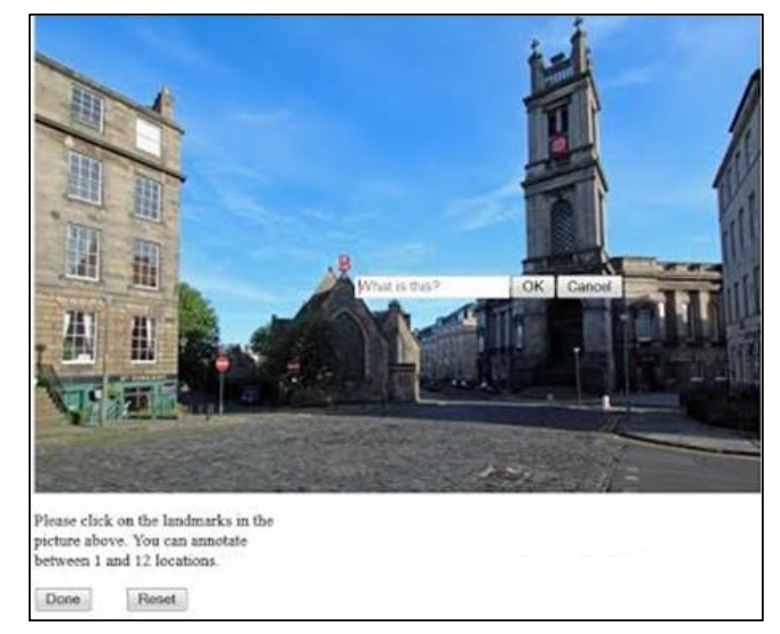

Figure 2: Web based landmark tagging experiment (a) overview and magnified region (b) adding annotation text 
difference noted between males and females in their choice of landmarks, or the number of landmarks identified per image. Figure $3 \mathrm{~b}$ shows how well the participants thought they knew Edinburgh, with most candidates having at least some knowledge of the region. There was a trend for males with better knowledge of the city to increase annotation length marginally, and for those familiar with the city to name landmarks. The number of tags per image ranged from 178 tags to 451 tags, with an average of 90 unique participants per image. The average number of tags generated per participant was 56, with a total of 10,350 tags 204 created across the 37 images.

a)

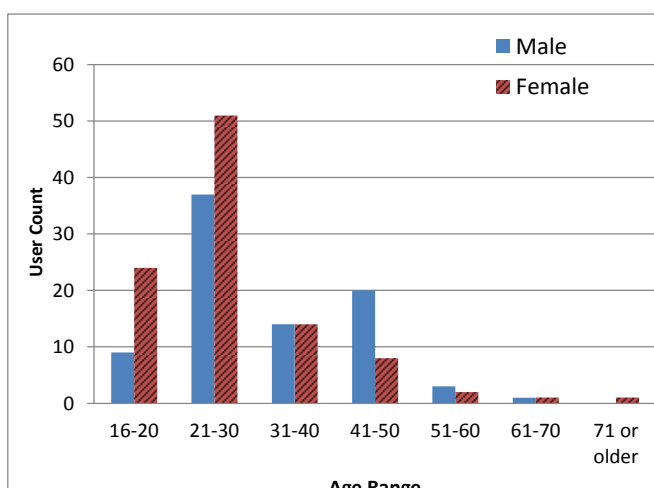

b)

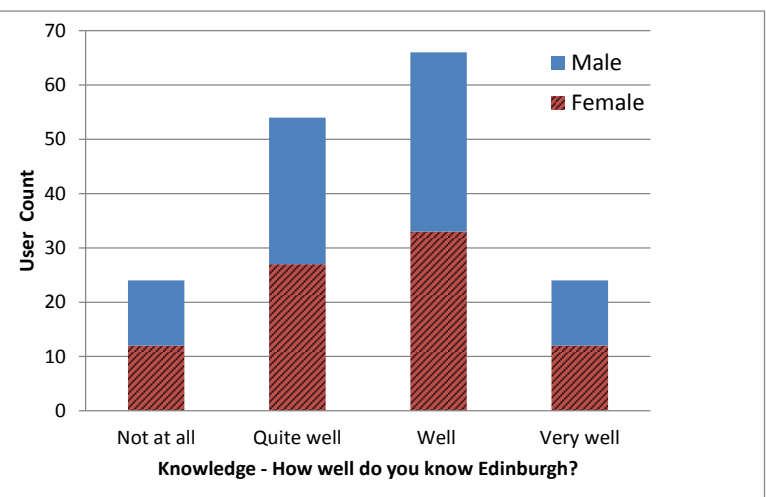

Figure 3: Breakdown of Data by Participant Group

(a) count of users (b) knowledge of the city

\section{Web Data Collection Results}

An example of the output is shown in Figure 4, which displays the tagged locations and the supplied tag text. The tags for this image are shown as a word cloud in Figure 5, whereby the more frequently occurring terms are represented in a larger font. In this example the tower and clock refer to the church on the right side of Figure 4, while church was used for both churches in the scene. Without considering the tag locations it is not possible to identify these two distinct groups from the term frequency (i.e. word cloud), nor therefore identify landmark dominance in the scene at a feature level.

Therefore analysis of the annotation text results alone was not suitable to identify landmark tag clusters because in each scene there may be multiple instances of a feature type (e.g. a small church and a large church with spire), and it would not be possible to rank individual object popularity based on term frequency.

The spatial pattern of the supplied tag locations may be summarised using spatial clustering, such as Kernel Density Estimation (KDE), (Silverman, 1986). The results for four example scenes are shown in Figure 6, where red shows a dense concentration of user tags. These dense spatial concentrations are clearly noticeable for the two churches and the building on the left of the scene (a public house) in Figure 6a. In particular there is a concentration of 
tags around the top of the taller church tower where there is a clock face. In Figure $6 \mathrm{~b}$ the KDE has highlighted a single cluster (Group 1) where there are in fact two distinct features, which are at different viewing distances but a similar viewing angle from the observer. Figure 7 shows this in greater detail where, due to the KDE bandwidth setting, groups (i) and (ii) have merged resulting in a dense cluster that does not depict a single landmark but is an artefact of two objects 180 metres apart having a similar viewing angle from the observer.

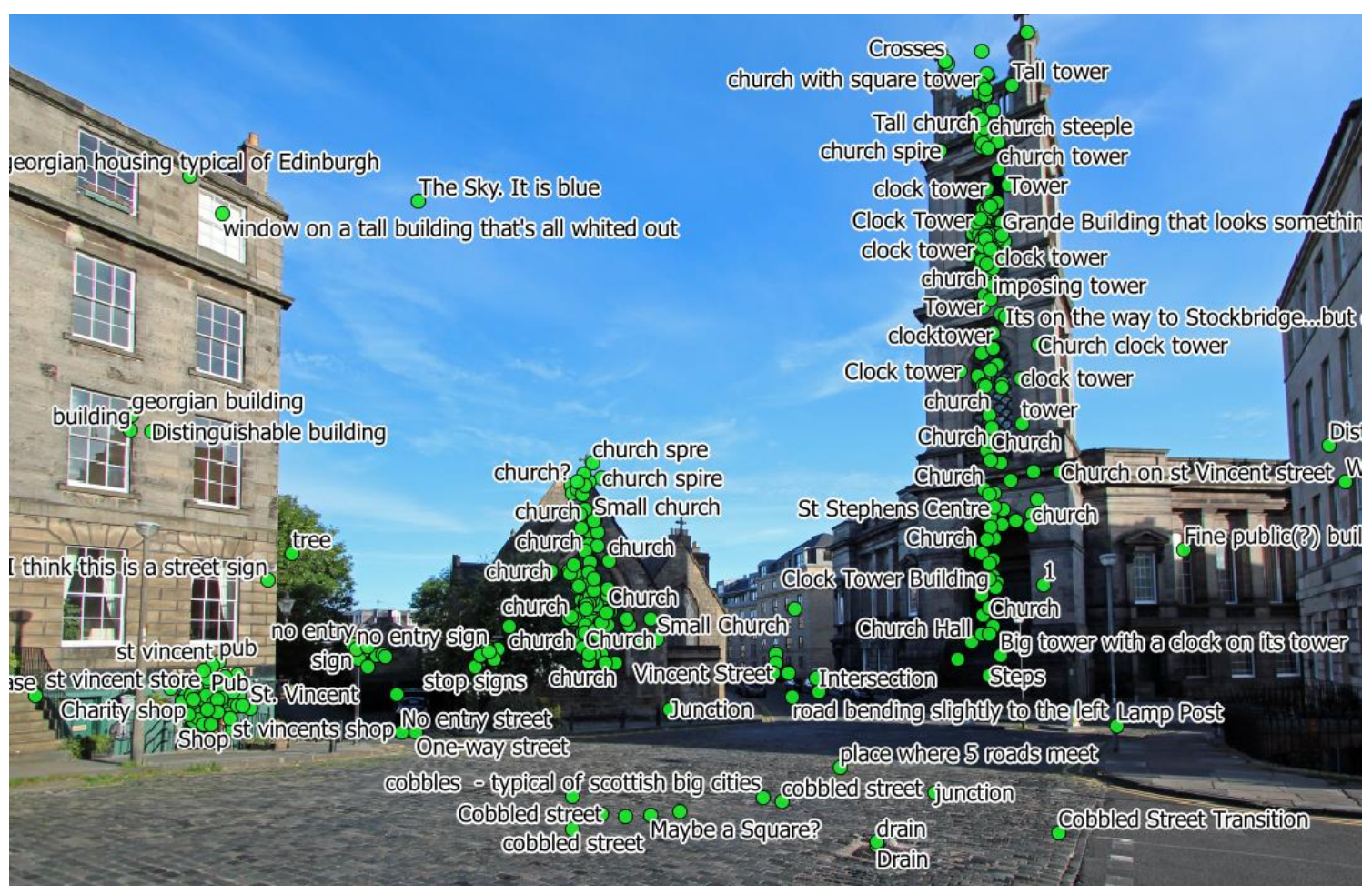

Figure 4: Example Scene with Some (not all) Tags from All Users Displayed

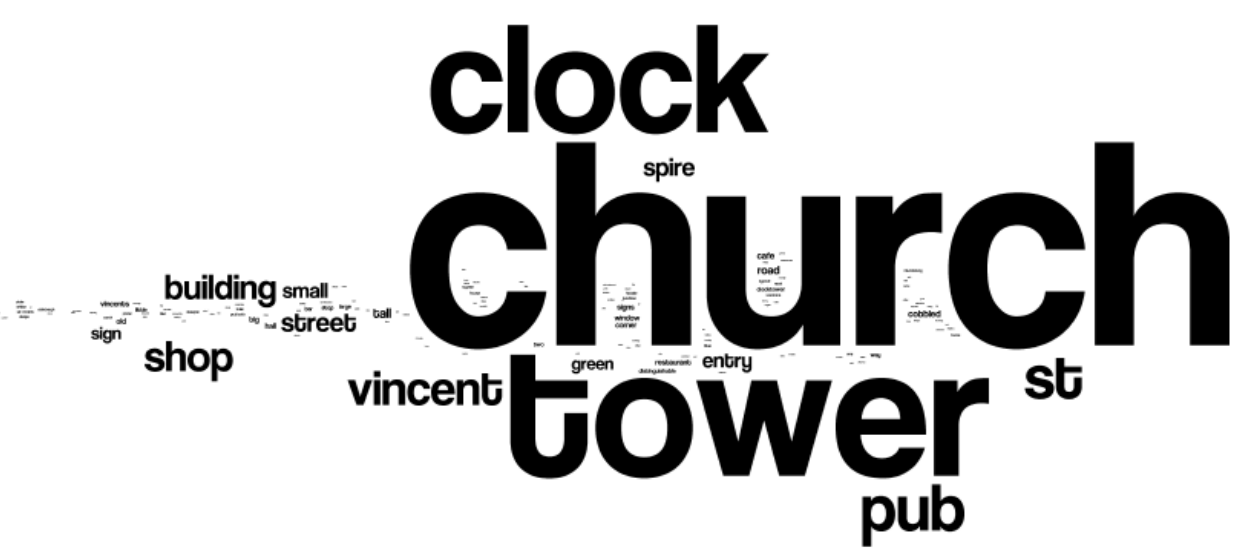

Figure 5: Word Cloud for Tags from a Single Image 
231 Group 4 in Figure 6d consists of tags for 'Scott Monument' and 'Calton Hill' which have 232 been clustered together despite being more than 670 metres apart, for the same reason of the 233 viewing angle. Similarly three objects at different viewing distances are clustered in Group 6.

234 In contrast Groups 2, 3 and 5, in Figure 6b, Figure $6 c$ and Figure $6 d$ respectively are 235 examples of single features yet each is presented as two distinct clusters. This is interesting 236 as it shows the participants considered the object to have multiple focal points of interest, 237 nevertheless it is necessary to aggregate these tag clusters in order to determine the 238 dominance of these features as single objects in the scene. 
a)

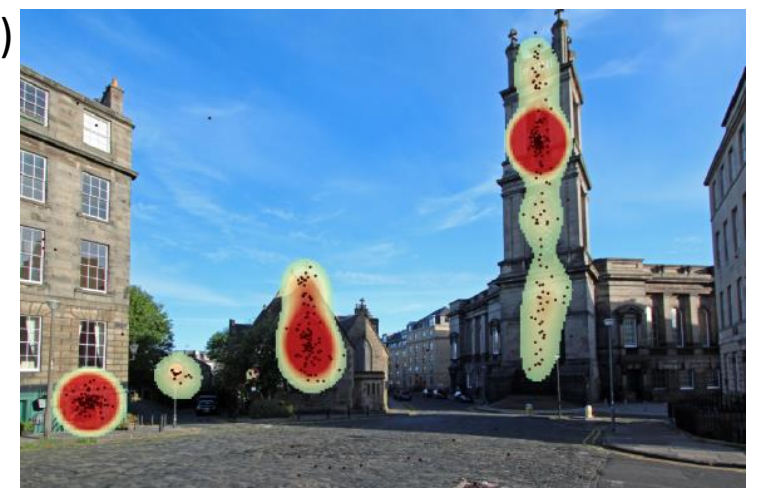

b)

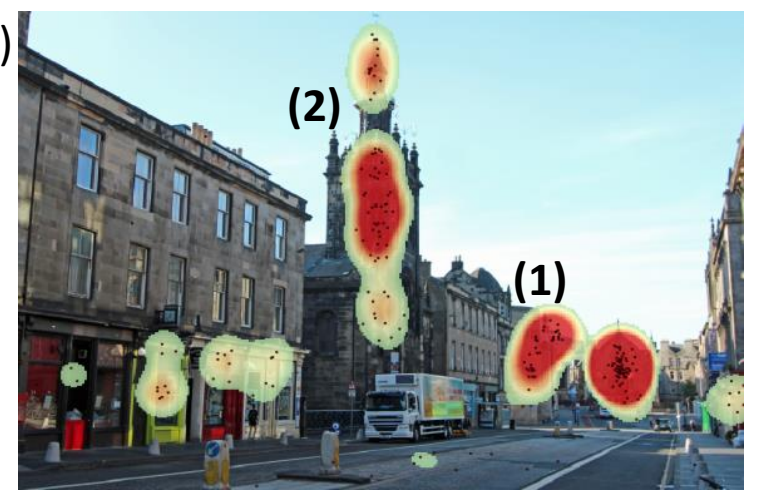

c)

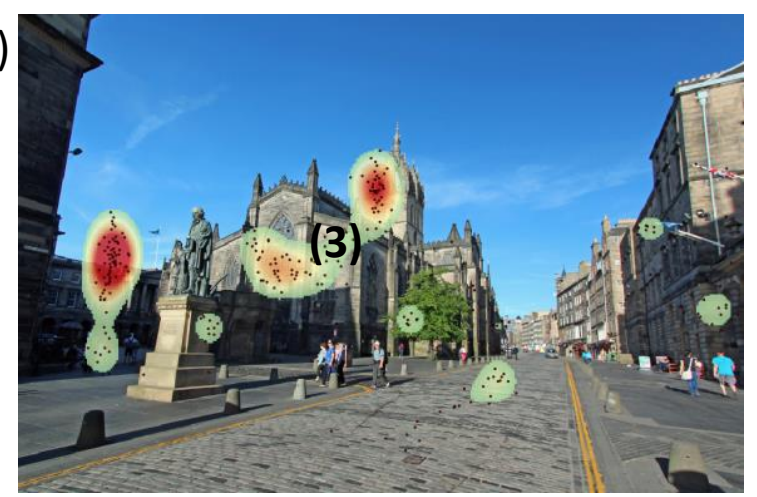

d)

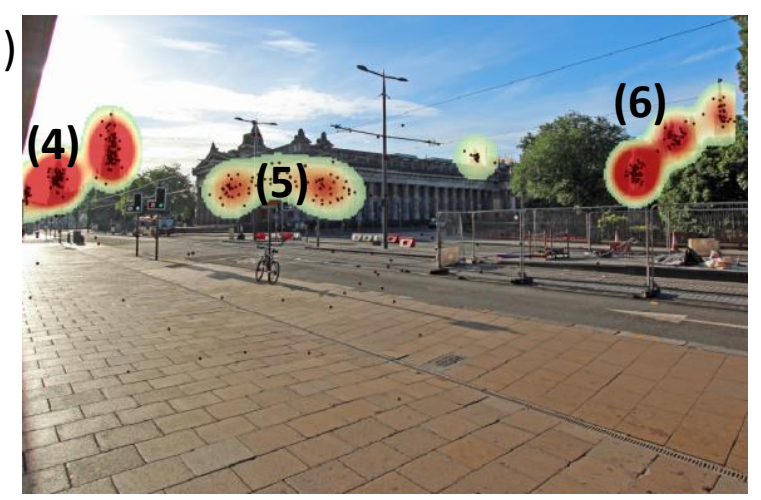

Figure 6: Kernel Density Estimation for User Tags (where red = dense clustering)

241 To improve upon this outcome a clustering technique was developed which included both 242 spatial and semantic components, as described in Section 5. The performance of this 243 approach is discussed in Section 6. 


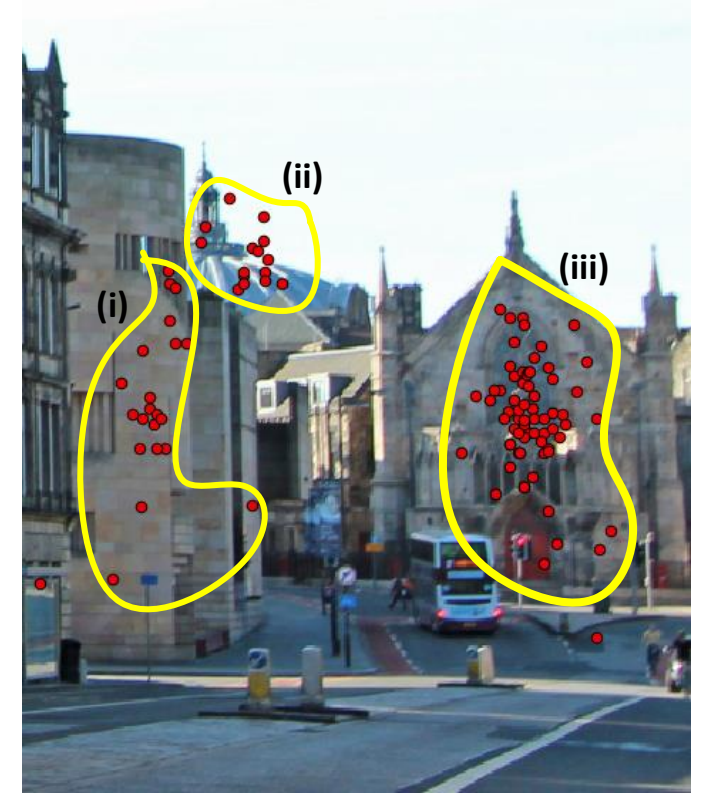

Figure 7: Spatial Clustering Errors due to not Modelling Distance

Group (i) and group (ii) tag locations should be considered separately as the objects are 180 metres apart

\section{5. Spatial and Semantic Clustering}

245 The participants supplied a text annotation for each tag, consisting of any number of words.

246 This allowed for the creation of a more natural dataset of descriptive object terms to be 247 collected, but added complexity in the analysis and term matching.

248 A fuzzy text matching technique based on character level trigrams was used to group similar 249 terms (Lin, 1998; Zamora, Pollock, \& Zamora, 1981). This rated phrase similarity by 250 calculating the number of shared three letter combinations found, while ignoring punctuation 251 and letter case. To improve the matching process it was necessary to also ignore stop words such as 'of', 'the', and ' $a$ '. The Trigram matching results are shown for a number of examples in Table 1, with values from 0 (no match) to 1 (exact match). Trigrams perform well in matching word stems ('church' versus 'churches'), and misspellings ('monument' vs 'momument'). However they are not able to recognise semantic similarities, for example the connection between a tag labelled church and another labelled cathedral (score of 0.0625), or match the Scottish word kirk with church (score of 0).

258 To improve this an enhanced matching function was developed which included access to a synonym table allowing for conceptually similar terms, such as 'street' and 'road', 'cathedral' and 'church', and 'memorial' and 'statue' to be treated as identical. The results are shown in Table 1, where 'church' and 'cathedral' score an exact match of 1.0, and 'church tower' and 'cathedral spire' also score an exact match. The synonym table was hand constructed by looking at the most commonly occurring words from all images. It would also be possible to populate such a table using an existing database of synonyms such as WordNet (Princeton University, 2010). However this enhanced matching function lacked the ability to model partonomic relationships (i.e. relationships between an object's parts) therefore the score for 'church' and 'clock tower' remained low (0.0556). 
To improve on this phrase pairs were collected by processing each tag in turn, searching the corresponding image for nearby tags within a defined pixel distance, equivalent to the KDE bandwidth. The content similarity score for each tag pair was calculated and those with a score greater than 0.3 , the default value for the trigram module used (Bartunov, Sigaev, \& Kings-Lynne, 2014), were considered to be related. All of the tags were processed resulting in a topological network of connected content for each image.

Table 1: Word Similarity using Trigrams

\begin{tabular}{l|l|l|l}
\hline \multicolumn{1}{c|}{ Phrase One } & \multicolumn{1}{|c|}{ Phrase Two } & \multicolumn{1}{c}{$\begin{array}{c}\text { Trigram Matching } \\
\text { (0 to 1) }\end{array}$} & \multicolumn{1}{c}{$\begin{array}{c}\text { Enhanced Matching } \\
\text { (0 to 1) }\end{array}$} \\
\hline $\begin{array}{l}\text { church } \\
\text { monument }\end{array}$ & $\begin{array}{l}\text { Churches } \\
\text { momument * }\end{array}$ & 0.6000 & 0.6000 \\
church & Cathedral & 0.5000 & 0.5000 \\
church tower & Clock & 0.0625 & 1.0000 \\
church tower & Cathedral & 0.0556 & 0.0556 \\
church tower & cathedral spire & 0.0455 & 0.5385 \\
church & kirk & 0.0357 & 1.0000 \\
church of St Giles & St Giles Kirk & 0.3750 & 1.0000 \\
\hline
\end{tabular}

\subsection{Expanding the Network of Linked Tags using a Secondary Pass}

In some cases running the process a single time resulted in small groups of tags being left as orphan clusters. For example in Figure 8 on the 'First Pass' three cluster groups were formed, relating to two objects; a no entry sign and a church with a clock tower. The two groups on the right remain distinct as no synonym entry links the church tags with clock or clock tower, and the other clock tower tag was outside the search radius. This can be addressed by increasing the search distance but that could result in separate object instances being combined (e.g. two nearby churches in Figure 4 would be grouped as a single entity). Instead the data was processed a second time using the same buffer distance but the vocabulary of related terms was increased by using the word lists generated from the first pass. By doing this the conceptual links list is automatically expanded for tag groups nearby allowing for greater conceptual links, but reducing the likelihood of separate objects being merged due to the limited spatial search parameters. This is a form of query expansion (Chum, Philbin, Sivic, Isard, \& Zisserman, 2007; Xu \& Croft, 1996), limited by the spatial location of the supplied tags. For example a church node may be joined to a clock tower node, even though they do not share any similar terminology based on a church tower node elsewhere being linked to a clock tower through the common term tower. Figure 8 shows an example of this process, where initially links are made between tags forming 3 networks based on common terminology. These network phrases are used during a second pass of the data, whereby a 
greater number of linkages may be added between groups as a result of the expanded semantic connections learned from the initial pass. The result is an expansion of the network topology through the linking of network groups, a reduction in the number of object groups, and an increase in linkages made between object parts thereby improving the partonmic modelling capability.

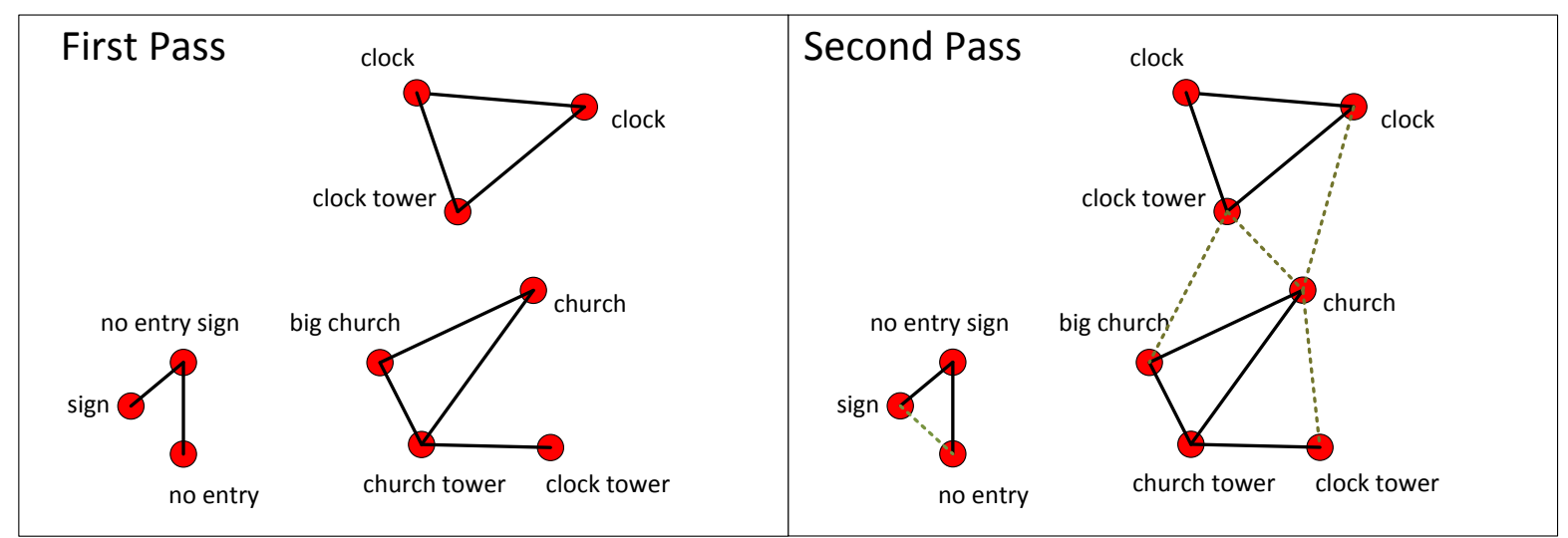

Figure 8: Expanding the Linked Network with Secondary Pass

\section{Tag Clustering Results}

An example of the output from this process is shown in Figure 9, where colours are assigned randomly based on Cluster Group ID. There are many improvements compared to the spatial only clustering (Figure 6), as now two groups are identifiable in Figure 9b (group 1) where before there was a single cluster, and a single group identified as group 2. Figure 9d (group

311 6) also now shows three distinct object definitions, rather than a single cluster.

312 Figure 9b (group2), Figure 9c (group 3), and Figure 9d (group 5) are now shown as single 313 objects rather than before where the variety of focal points selected to tag the object by the participants had resulted in multiple cluster centres on these objects. The previously single group at d (group 4) is now separated into two groups, however there is also an overlap occurring (orange group connects to red group) which is due to a common use of

317 terminology ('tower'). 
a)

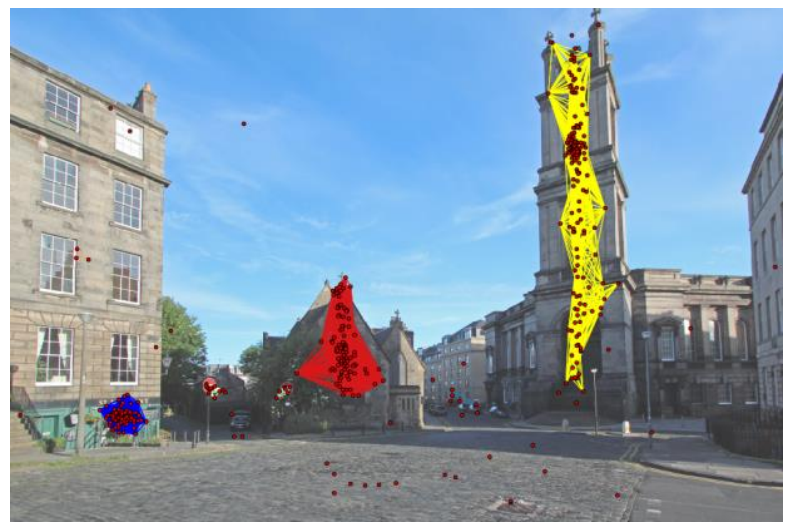

b)

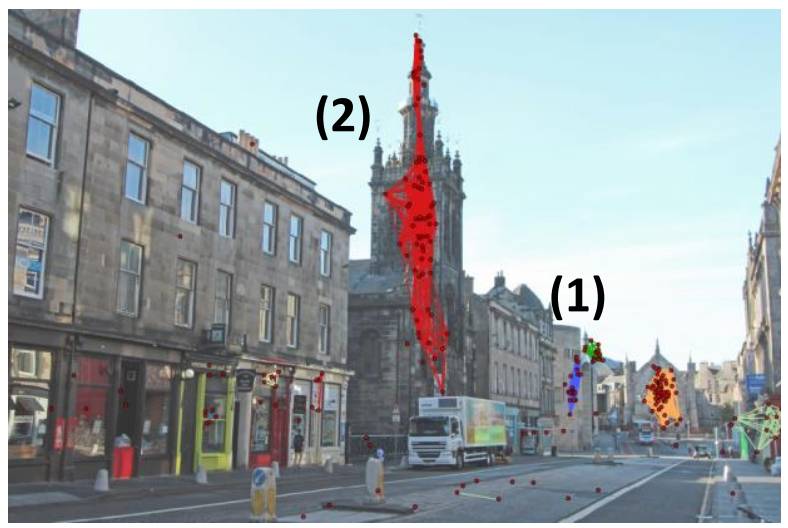

c)

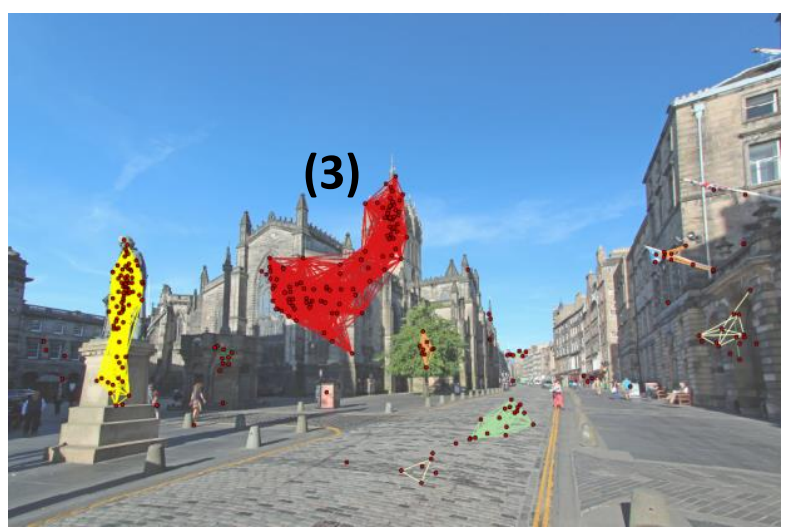

d)

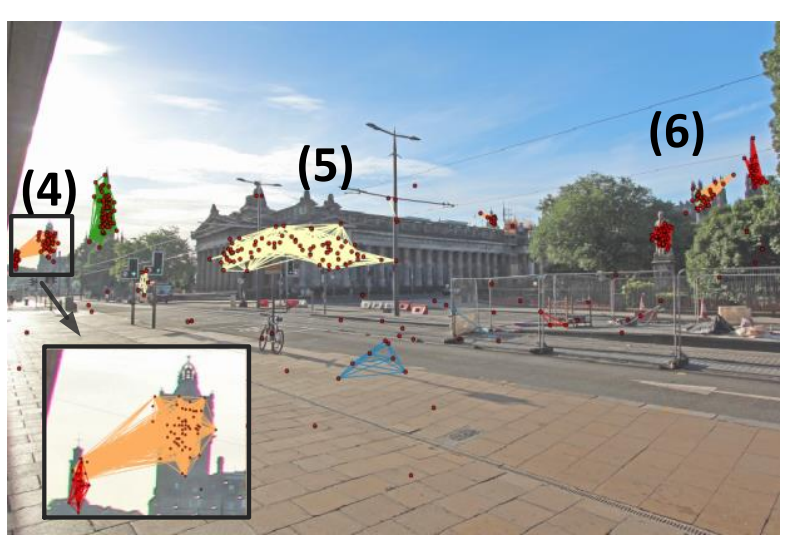

Figure 9: Spatial-Semantic Clusters (random cluster colouring) 
Tag groups were identified for each image making it possible to automatically generate related word lists. For example church is linked to church spire, which is linked to church tower, which is linked to clock tower, which is linked to clock. Tags define objects spatially and conceptually, and the frequency of each tagged phrase gives an indication of the most common term used for that object and its parts. Once tags have been linked in this way it is possible to calculate tag group centroids relating to the concept centres, for example the clock on the clock tower, which is part of the church. It is also possible to generate a list of the most frequent terms used per object, rather than per image, as shown in Figure 10.

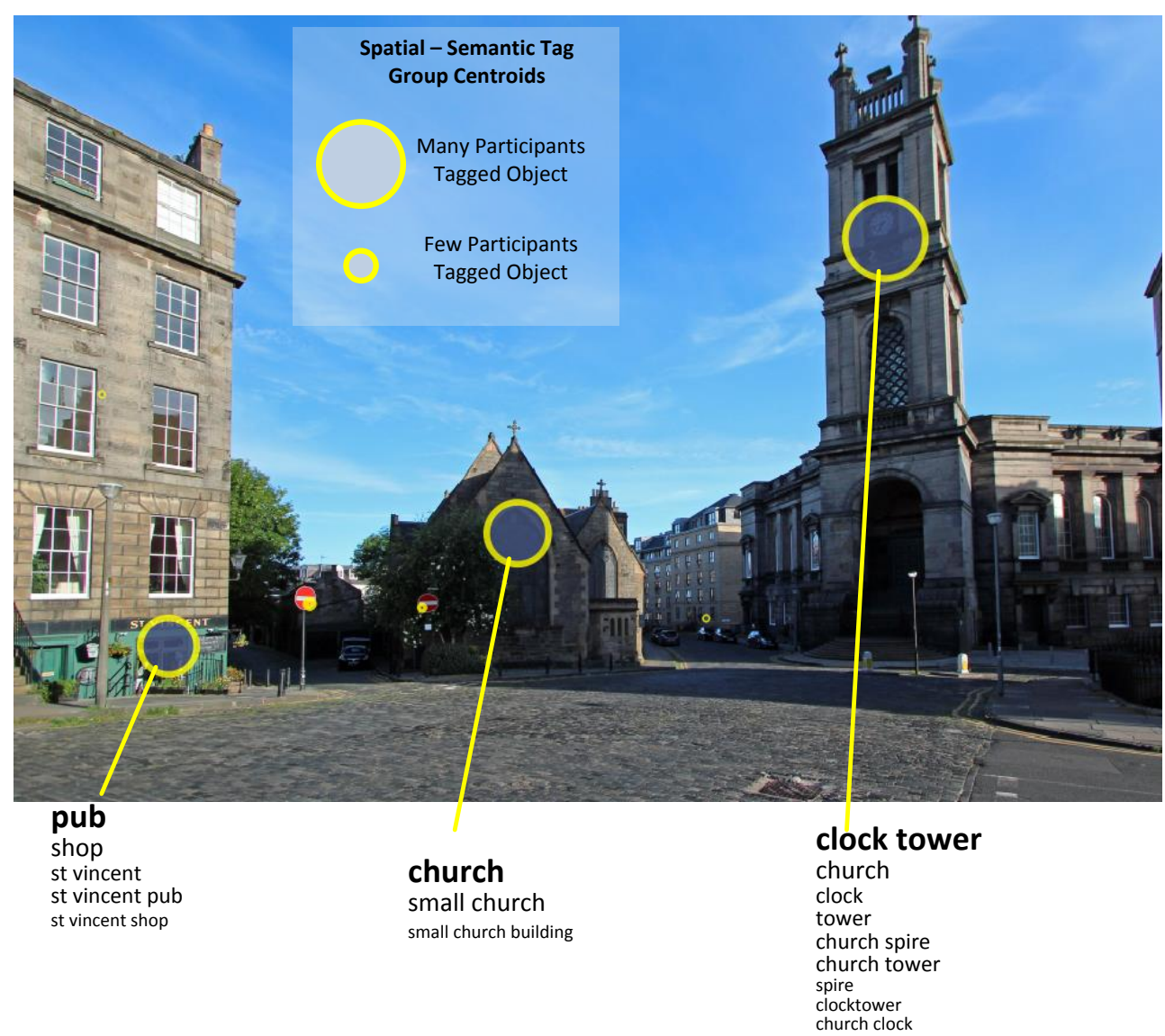

Figure 10: Phrase Ranking per Identified Object

Comparing the spatial clusters against the spatial-semantic clusters gives an insight into objects which are interesting and easy to define versus those of interest which are hard to define. 
The strength of links between all tags in an image can be calculated as a function of phrase similarity and the inverse tag distance, such that similar phrases near each other receive a high value. Figure 11 visualises this for the example scenes, where very strong relationships are shown in black, strong links in blue, and weak links in purple. A low threshold was specified to remove links between very unrelated tags. This visualises the conceptual connections between features.

The object concept centres, as introduced in Figure 10, are displayed as yellow dots to indicate the main identified features in each example (Figure 11, right column). The weak links, shown in purple, can be considered as mapping objects which might be confused from having similar annotations. For example the link between the two churches in (a) and (c) shows their conceptual similarity and highlights the risk of a misunderstanding occurring if attempting to identify the object from the annotation alone. Also in (b) the "spire", "gallery", and "park" labels indicate objects which could be confused unless further details are included in a referring expression.

a)

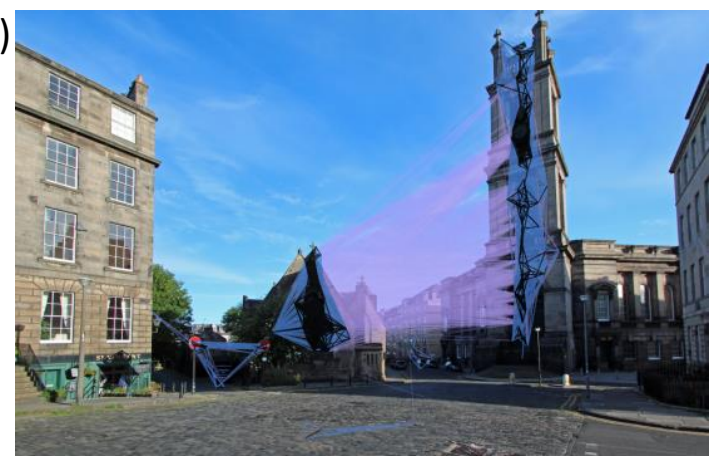

b)

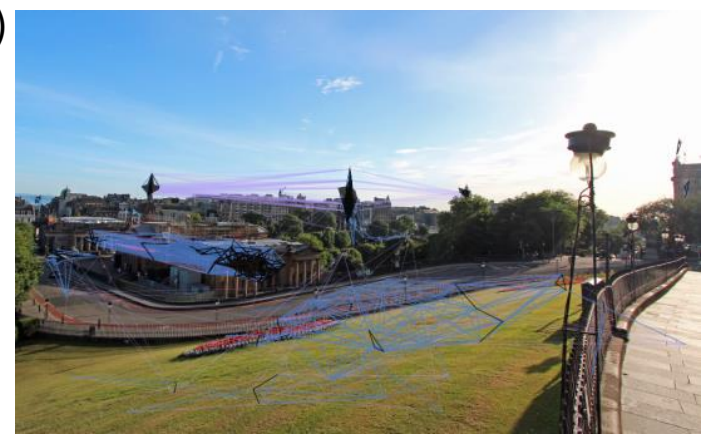

c)

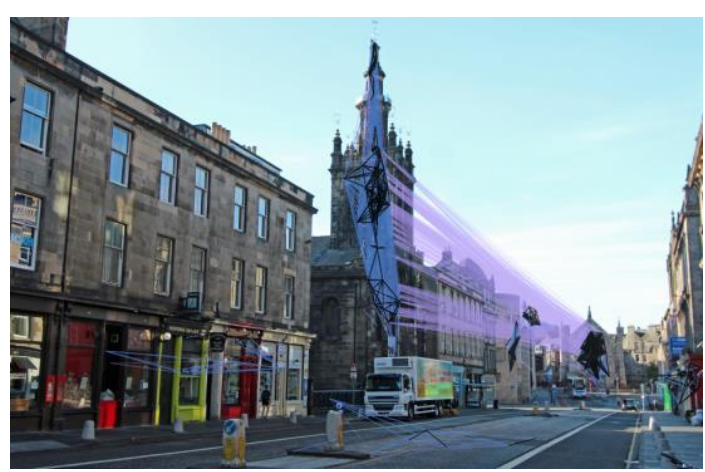

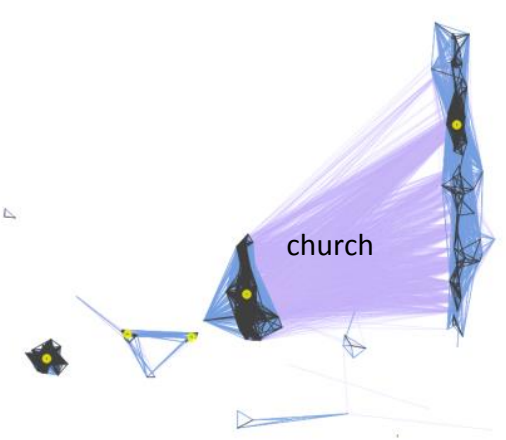
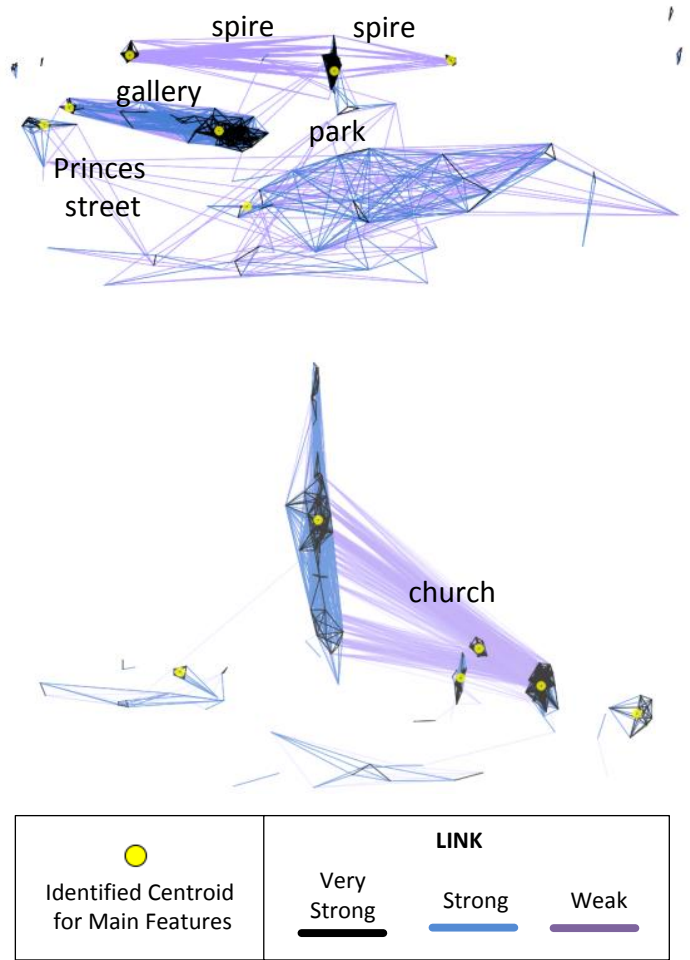

Figure 11: The Strength of the Linkages between Tags 
The "Princes Street" link shown in Figure 11b has arisen from a mistake made by a number of participants who believed the foreground grassy region to be "Princes Street Gardens", and therefore the clustering method has calculated a weak link from these tags to "Princes Street" in the distance. While it is not possible to automatically discount these incorrect tags, it is possible to validate the strength of their linkage through the annotation matching, and distance calculations. As this example shows the link strength is considered to be weak, and effectively removes these tags from the calculation of object concept centres.

\section{Outstanding Issues and Future Work}

358 Overall the clustering technique performed well in reducing the supplied tags to related groups representing single objects in the scene, but there were cases that raised some outstanding issues. These fell into two categories: cases where two nearby objects were described in a similar way but were in fact unrelated, and where an object was described in two very different ways resulting in their being no semantic overlap.

Figure 12(a) shows an example of the first category, where three object clusters are identifiable but an incorrect link has been made between objects (i) and (ii). This occurred because some participants described the first object (i) as a tower, while others described object (ii) as a steeple. These words were linked together via an entry in the synonym table, and could be highlighted for checking, but to automate this disambiguation will require further work.

Another issue was that it was difficult to associate the proper noun with a landmark description. For example in Figure 12(b), some familiar with the city labelled "Scott Monument" while others labelled it as "Steeple". These two concepts do not have a semantic link and therefore the output shows two overlapping groups, where there should be a single entity. These can be automatically highlighted by using spatial containment functions to produce a list of such co-occurrences to be examined in more detail and resolved by adding an entry into the synonym table to link the groups.

a)

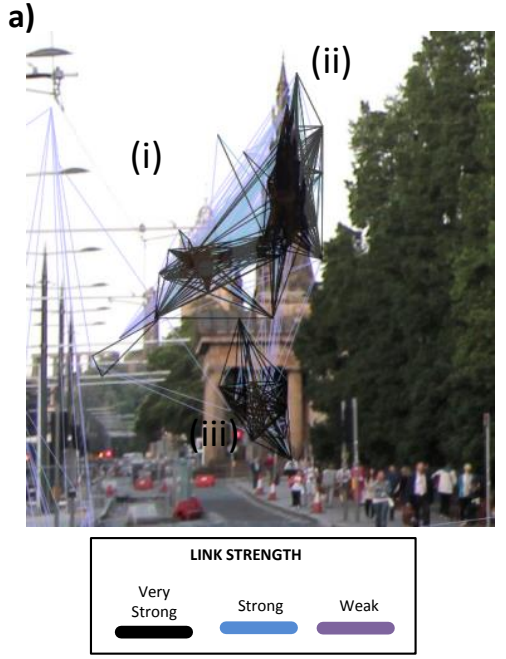

b)

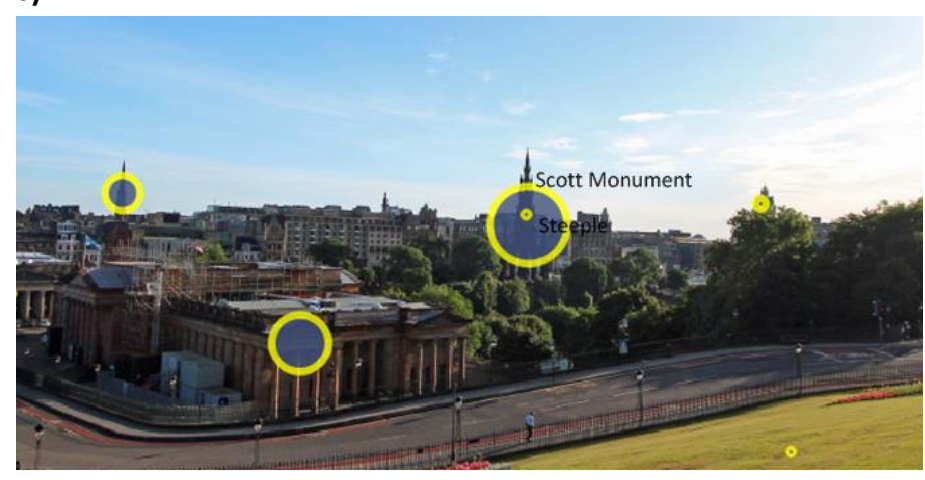

Figure 12: Examples of Outstanding Issues

(a) Linkage Strengths between Tags (b) Cluster Group Centroids 


\section{Conclusions}

381 The paper outlines a method to identify clusters of tags supplied for urban scenes. A web 382 based experiment was conducted whereby people tagged objects they considered to be 383 interesting in the urban scene, adding free text annotations. The dataset was analysed to 384 identify the interesting city objects in each image. Spatial clustering alone was shown to be 385 flawed in certain cases where two objects are at a similar viewing angle, but different 386 distances away from the observer. Instead a new method was developed which combined spatial and semantic clustering techniques.

The method collects nearby tags which show a correlation using trigram fuzzy matching. Synonyms and stop words were used to improve the matching, and a network graph of connected tags was generated for each image. This was expanded in a secondary pass by using the linkages discovered on the first pass to join up orphaned tag groups. The results show that it was possible to automatically identify objects of interest from the user supplied tags, and that term frequencies could be discovered at an object level. The network graph visualises the relationships which exist between tags, enabling the strength of the relations to be inferred from the density and centrality of the graph edges.

This research has relevance in the context of intuitive dialogue driven systems in which rich descriptions of landmarks are required to support the generation of way finding instructions (Kai-Florian Richter, Tomko, \& Winter, 2008) since the graph is able to provide both a generic description ('the church') for the observer in the far distance, and a detailed description ('the church tower with the clock') when the observer is closer. The next phase of this research is to compare the results of this user experiment against a model of landmark saliency, whereby the relative dominance of landmarks selected from this study will be compared at an object level with the saliency model output. Where differences are noted the input weightings of saliency model parameters (e.g. visible area, on the skyline, viewing distance, object type) will be adjusted to more closely match these findings from this experiment. Term frequencies and variations by object type and viewing distance will be conducted, giving a greater understanding of how people refer to features of interest in urban scenes which could then be incorporated into the natural language generation component.

\section{9. Acknowledgements}

412 The research leading to these results has received funding from the EC's 7th Framework 413 Programme (FP7/2011-2014) under grant agreement no. 270019 (SpaceBook project).

\section{$416 \quad$ 10.References}

417 Ahern, S., Naaman, M., Nair, R., \& Yang, J. (2007). World explorer: Visualizing aggregate data from unstructured text in geo-referenced collections In JCDL (pp. 1--10).

Bartie, P., \& Mackaness, W. A. (2006). Development of a speech-based augmented reality 
system to support exploration of cityscape. Transactions in GIS, 10(1), 63-86. doi: 10.1111/j.1467-9671.2006.00244.x

Bartunov, O., Sigaev, T., \& Kings-Lynne, C. (2014). Additional Supplied Modules (pg_trgm). (12 May 2014). http://www.postgresql.org/docs/9.1/static/pgtrgm.html

Caduff, D., \& Timpf, S. (2008). On the assessment of landmark salience for human navigation. Cognitive Processing, 9(4), 249-267.

Chen, G., \& Kotz, D. (2000). A survey of context-aware mobile computing research: Technical Report TR2000-381, Dept. of Computer Science, Dartmouth College.

Chum, O., Philbin, J., Sivic, J., Isard, M., \& Zisserman, A. (2007). Total recall: Automatic query expansion with a generative feature model for object retrieval. Paper presented at the Computer Vision, 2007. ICCV 2007. IEEE 11th International Conference on.

Daley, T. (2012). We're now officially in the Post-PC era. Waste + Water Management Australia, Vol. 38(No 6), 27-30.

Daniel, T. C., \& Vining, J. (1983). Methodological Issues in the Assessment of Landscape Quality. In I. Altman \& J. Wohwill (Eds.), In Behaviour and the Natural Environment (pp. 39-83). New York: Plenum Press.

Duckham, M., Winter, S., \& Robinson, M. (2010). Including landmarks in routing instructions. Journal of Location-Based Services, 4 (1), 28-52.

Elias, B. (2003). Extracting landmarks with data mining methods. In W. Kuhn, M. F. Worboys, \& S. Timpf (Eds.), Spatial information theory (Vol. 2825, pp. 398-412). Berlin: Springer.

Elias, B., \& Brenner, C. (2004). Automatic generation and application of landmarks in navigation data sets. In P. F. Fisher (Ed.), Developments in Spatial Data Handling (pp. 469-480). Berlin: Springer.

Grice, H. P. (1975). Logic and conversation. In P. Cole \& J. Morgan (Eds.), Syntax and Semantics 3: Speech Acts (pp. 41-58): Academic Press.

Hirtle, S. C., \& Heidorn, P. B. (1993). The structure of cognitive maps: Representations and processes. Behavior and Environment: Psychological and Geographical Approaches, 170-192.

Hirtle, S. C., \& Jonides, J. (1985). Evidence of hierarchies in cognitive maps. Memory and Cognition, 13(3), 208-217.

Ishii, H., Kobayashi, M., \& Arita, K. (1994). Iterative design of seamless collaboration media. Communications of the ACM, 37(8), 83-97.

Lee, J. (2014). Project Tango. Retrieved 6 June 2014, from https://www.google.com/atap/projecttango

Lin, D. (1998, July 24-27). An information-theoretic definition of similarity. Paper presented at the Proceedings of the Fifteenth International Conference on Machine Learning, Madison, Wisconsin, USA.

Linton, D. L. (1968). The assessment of scenery as a Natural Resource. Scottish Geographical Magazine, 84, 219 - 238.

Liu, D., Hua, X.-S., \& Zhang, H.-J. (2011). Content-based tag processing for internet social images. Multimedia Tools and Applications, 51(2), 723-738. 
Long, S., Aust, D., Abowd, G., \& Atkeson, C. (1996). Cyberguide: prototyping contextaware mobile applications. Paper presented at the Conference on Human Factors in Computing Systems, Vancouver.

Lovelace, K. L., Hegarty, M., \& Montello, D. R. (1999). Elements of good route directions in familiar and unfamiliar environments. In C. Freksa \& D. Mark (Eds.), Spatial Information Theory: Cognitive and Computational Foundations of Geographic Information Science (Vol. 1661, pp. 751): Springer Berlin / Heidelberg.

Mackaness, W. A., Bartie, P., Dalmas, T., Janarthanam, S., Lemon, O., Liu, X. (2014, 7-13 April). Talk the Walk and Walk the talk: Design, Implementation and Evaluation of a Spoken Dialogue System for Route Following and City Learning. Paper presented at the Annual Conference of the Association of American Geographers, Tampa Florida.

Noh, H.-Y., Lee, J.-H., Oh, S.-W., Hwang, K.-S., \& Cho, S.-B. (2012). Exploiting indoor location and mobile information for context-awareness service. Information Processing \& Management, 48(1), 1-12.

Nothegger, C., Winter, S., \& Raubal, M. (2004). Computation of the Salience of Features. Spatial Cognition and Computation, 4(2), 113-136. doi: 10.1207/s15427633scc0402_1

Papadopoulos, S., Zigkolis, C., Kompatsiaris, Y., \& Vakali, A. (2010). Cluster-based landmark and event detection on tagged photo collections. IEEE Multimedia.

Princeton University. (2010). WordNet. (5 May 2014). wordnet.princeton.edu

Raubal, M., \& Winter, S. (2002). Enriching wayfinding instructions with local landmarks In M. J. Egenhofer \& D. M. Mark (Eds.), Second International Conference GIScience (Vol. 2478, pp. 243-259). Boulder, USA: Springer.

Richter, K.-F., Tomko, M., \& Winter, S. (2008). A dialog-driven process of generating route directions. Computers, Environment and Urban Systems, 32(3), 233-245.

Richter, K.-F., \& Winter, S. (2014). Landmarks: Springer International Publishing.

Shafer, E. L., \& Brush, R. O. (1977). How to measure preferences for photographs of natural landscapes. Landscape Planning, 4, 237-256.

Silverman, B. W. (1986). Density estimation for statistics and data analysis (Vol. 26): CRC press.

Tousch, A.-M., Herbin, S., \& Audibert, J.-Y. (2012). Semantic hierarchies for image annotation: A survey. Pattern Recognition, 45(1), 333-345.

Tversky, B. (1993). Cognitive maps, cognitive collages, and spatial mental models. Paper presented at the Spatial Information Theory: A Theoretical Basis for GIS, Italy.

Vonikakis, V., Jinda-Apiraksa, A., \& Winkler, S. (2014). PhotoCluster: A Multi-clustering Technique For Near-duplicate Detection In Personal Photo Collections. Paper presented at the VISAPP, Lisbon, Portugal.

Wang, M., Ji, D., Tian, Q., \& Hua, X.-S. (2012). Intelligent photo clustering with user interaction and distance metric learning. Pattern Recognition Letters, 33(4), 462-470.

Weiser, M., Gold, R., \& Brown, J. S. (1999). Origins of ubiquitous computing research at PARC in the late 1980s. IBM Systems Journal, 38(4), 693-695.

Werner, S., Krieg-Bruckner, B., Mallot, H. A., Schweizer, K., \& Freksa, C. (1997). Spatial 
ognition: The role of landmark, route, and survey knowledge in human and robot navigation. In M. Jarke (Ed.), Informatik '97 GI Jahrestagung (pp. 41-50 ). Berlin, Heidelberg, New York. Springer.

Winter, S., Tomko, M., Elias, B., \& Sester, M. (2008). Landmark hierarchies in context. Environment and Planning B: Planning and Design, 35(3), 381 - 398.

Xu, J., \& Croft, W. B. (1996). Query expansion using local and global document analysis. Paper presented at the Proceedings of the 19th annual international ACM SIGIR conference on Research and development in information retrieval.

Zamora, E. M., Pollock, J. J., \& Zamora, A. (1981). The use of trigram analysis for spelling error detection. Information Processing \& Management, 17(6), 305-316.

517 Zube, E. H., Sell, J. L., \& Taylor, J. G. (1982). Landscape perception: research, application and theory. Landscape Planning, 9(1), 1-33. 